\title{
Mathematical Modeling of a Supply Chain with Uncertain Parameters
}

\section{Raad Alzahrani}

National Water Company, Saudi Arabia

Correspondence Author: Raad Alzahrani, National Water Company, Saudi Arabia

Received date: 12 June 2018, Accepted date: 4 September 2018, Online date: 13 September 2018

Copyright: (C) 2018 Raad Alzahrani, This is an open-access article distributed under the terms of the Creative Commons Attribution License, which permits unrestricted use, distribution, and reproduction in any medium, provided the original author and source are credited.

\begin{abstract}
Decision making in supply chain network problems is fraught with numerous uncertainties. In this paper, we discuss and illustrate a four-layer supply chain network including suppliers, manufacturers, warehouses, and markets, which is designed to address the production and transportation of a single product. We present a robust optimization model that is useful in analyzing uncertainties associated with the primary input parameters including demand, supply, and cost parameters since it does not affect the complexity of the original deterministic model. The objective of the mathematical model is to obtain the optimal location of manufacturers and warehouses as well as the quantities of raw materials and final products, which result in the minimum total costs including the penalty costs.
\end{abstract}

Key words: Supply Chain Cost, Robust Optimization, Uncertain Parameters, Degree of Conservatism.

\section{INTRODUCTION}

The mathematical formulation of the supply chain models have long been subject of academic studies. The primary objectives of supply chain models are maximizing service levels (Namdari et al., 2017), minimizing transit times, and minimizing the total costs including those of locating the manufacturers and warehouses, transportation of goods and products between the layers of a supply chain, etc. Hence, it is important to present an appropriate mathematical model (Tohidi, et al. 2017), which results in the minimum costs. Supply chain management with consideration of conflicting interests of the participants in the supply chain is very important for academia and industry (Yue\&You, 2014). Supply chain management can be categorized into three primary themes: purchasing, manufacturing, and transportation. Transportation is one of the main areas of supply chain management and has a significant impact on the profitability of companies. Transpiration as one of the main components of supply chain management, which refers to the movement of raw materials as well as the finished products between all the elements of a supply chain, needs to be cost-effective in order to ensure an efficient supply chain management (Vidal \&Goetschalckx, 2001).

Appropriate scheduling, planning and control policies must be used in order to optimize a supply chain. However, this optimization is subject to certain constraints and involves inherent uncertainties. There are different methodologies to model the uncertainties, including fuzzy base rules, possibility theory, and interval analysis (Stadtler, 2015), among which robust optimization approach has been of great academic interests especially with the recent improvements in computer science and simulation techniques.

Robust Optimization Procedure:

In this section, in order to clarify the model, we provide a brief introduction to robust optimization procedure introduced by Bertsimas and Sim (2004). Consider the following uncertain linear model:

$\operatorname{Min} \sum_{i, j} c_{i j} x_{j}$

S.t.: $\quad \sum_{i} \tilde{a}_{i j} x_{j} \geq b_{i} \quad \forall i$

$x_{j} \geq 0 \quad \forall j$

Assuming a nominal value $a_{i j}$ and a deviation from the nominal value $\hat{a}_{i j}$, the uncertain parameter $\tilde{a}_{i j}$ takes values in the interval $\left[a_{i j}-\hat{a}_{i j}, a_{i j}+\hat{a}_{i j}\right]$. The uncertainty budget parameter $\Gamma_{j}$ adjusts the robustness against the level of conservatism.

Therefore, model (1) is transformed into the following nonlinear model:

Minc' $\boldsymbol{x}$

S.t: $\quad \sum_{j} a_{i j} x_{j}-\underset{\left\{S_{i} \cup\left\{t_{i}\right\}\left|S_{i} \subseteq J_{i},\right| S_{i} \mid=\left\lfloor\Gamma_{i}\right\rfloor, t_{i} \in J_{i} \backslash S_{i}\right\}}{\max }\left\{\sum_{j \in S_{i}} \hat{a}_{i j} x_{i}+\left(\Gamma_{i}-\left\lfloor\Gamma_{i}\right\rfloor\right) \hat{a}_{i t_{i}} x_{j}\right\} \geq b_{i} \forall i$

$x_{j} \geq 0 \quad \forall j$

Let $\boldsymbol{x}^{*}$ be the optimal solution of the objective function, the protection function as a linear optimization problem is written as follows:

$\beta_{i}\left(\boldsymbol{x}^{*}, \Gamma_{i}\right)=\max \left\{\sum_{j \in J_{i}} \hat{a}_{i j} x_{j}^{*} w_{i j}\right\}$ 
$\sum_{j \in J_{i}} w_{i j} \leq \Gamma_{i}$

$0 \leq w_{i j} \leq 1 \quad \forall j \in J_{i}$

Therefore, model (1) can be rewritten as the following robust counterpart:

$\operatorname{Min} \sum_{j} c_{j} x_{j}$

S.t.: $\quad \sum_{j} a_{i j} x_{j}-\lambda_{i} \Gamma_{i}-\sum_{j \in J_{i}} \mu_{i j} \geq b_{i} \quad \forall i$

$\lambda_{i}+\mu_{i j} \geq \hat{a}_{i j} x_{j} \quad \forall i, j \in J_{i}$

$\mu_{i j} \geq 0 \quad \forall i, j \in J_{i}$

$\lambda_{i} \geq 0 \quad \forall i, x_{j} \geq 0 \quad \forall j$

where $\lambda_{i}$ and $\mu_{i j}$ are dual auxiliary variables.

Deterministic Model Formulation:

In order to facilitate the transportation between the suppliers and consumers and minimize the total costs of the supply chain network, the variables and objectives are transformed into a mathematical model. The supply chain network in this paper includes suppliers, manufacturers, warehouses, and markets as it is illustrated in Exhibit 1. The objective of this supply chain network is to determine the optimal quantity of raw materials shipped from suppliers to manufacturers, the quantity of products shipped from manufacturers to warehouses, and the quantity of products shipped from warehouses to markets, as well as location of manufacturers and warehouses so that the total costs will be minimized.

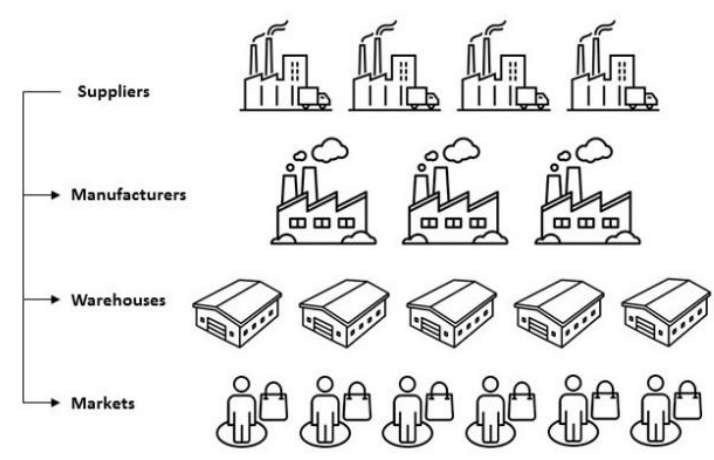

Exhibit 1: Structure of the supply chain network.

The following notation is used in the model:

Indices

$g \quad$ Index of potential suppliers

a Index of potential manufacturers

$b \quad$ Index of potential warehouses

$q \quad$ Index of potential markets

Parameters

$d_{Q} \quad$ Demand of market $q$

$k_{a} \quad$ Capacity of manufacturer $a$

$s_{g} \quad$ Capacity of supplier $g$

$w_{b} \quad$ Capacity of warehouse $b$

$t_{a}^{T} \quad$ Fixed cost of locating manufacturer $a$

$t_{b}^{W} \quad$ Fixed cost of locating warehouse $b$

$c_{g a}^{S T}$ Cost of shipping a unit of raw material from supplier $g$ to manufacturer $a$

$c_{a b}^{T W}$ Cost of shipping a unit of product from manufacturer $a$ to warehouse $b$

$c_{b q}^{W M}$ Cost of shipping a unit of product from warehouse $b$ to market $q$

$L_{q} \quad$ Unit penalty cost due to unsatisfied demand of markets $q$

$\alpha_{a} \quad$ Coefficient of raw material consumption in manufacturer $a$

$\beta_{b} \quad$ Coefficient of product stored in warehouse $b$

Decision variables

$$
\begin{aligned}
& y_{a}=\left\{\begin{array}{cc}
1 & , \text { if a manufacturer is located at } a \\
0, & \text { otherwise }
\end{array}\right. \\
& z_{b}=\left\{\begin{array}{cc}
1 & , \text { if a warehouse is located at } b \\
0, & \text { otherwise }
\end{array}\right.
\end{aligned}
$$

$x_{g a}$ Quantity of raw materials shipped from supplier $g$ to manufacturer $a$

$u_{a b}$ Quantity of products shipped from manufacturer $a$ to warehouse $b$

$v_{b q} \quad$ Quantity of products shipped from warehouse $b$ to market $q$

$A_{q} \quad$ Quantity of unsatisfied demands of market $q$

It terms of the above notation, the supply chain design problem can be formulated as follows:

$\operatorname{MinZ}=\sum_{a} t_{a}^{T} y_{a}+\sum_{b} t_{b}^{W} z_{b}+\sum_{g} \sum_{a} c_{g a}^{S T} x_{g a}+\sum_{a} \sum_{b} c_{a b}^{T W} u_{a b}+\sum_{b} \sum_{q} c_{b q}^{W M} v_{b q}+\sum_{q} L_{q} A_{q}$ 
The objective function (16) minimizes the penalty costs, the costs of locating factories and warehouses, and the shipment costs including those of raw materials and products.

$\sum_{a} x_{g a} \leq s_{g} \quad \forall g$

$d_{q}-\sum_{b} v_{b q} \leq A_{q} \quad \forall q$

$\sum_{b} u_{a b} \leq k_{a} y_{a} \quad \forall a$

$\sum_{q} v_{b q} \leq w_{b} z_{b} \quad \forall b$

$\sum_{g} x_{g a}=\alpha_{a} \sum_{b} u_{a b} \quad \forall a$

$\sum_{a} u_{a b}=\beta_{b} \sum_{q} v_{b q} \quad \forall b$

$x_{g a} \geq 0 \quad \forall g, a$

$u_{a b} \geq 0 \quad \forall a, b$

$v_{b q} \geq 0 \quad \forall b, q$

$A_{q} \geq 0 \quad \forall q$

Constraints (17) and (18) are supply and demand restrictions, respectively. Constraints (19) and (20) are manufacturer and warehouse capacity restrictions, respectively. Constraint (21) ensures that the quantity of raw materials shipped from the suppliers to a manufacturer equates the quantity of products shipped from the manufacturer to warehouses. Constraint (22) assures that the quantity of products shipped from the factories to a warehouse equates the quantity of products shipped from the warehouse to the markets. Constraints (23)-(26) enforce the non-negativity restrictions on corresponding decision variables.

\section{Robust Model Formulation:}

This section introduces a robust model when uncertainties are applied to cost of locating the manufacturers, cost of locating the warehouses, shipment costs, demand of the markets, and supply capacity of the suppliers. To specify the study scope, two assumptions and simplifications are postulated in the proposed model formulation as follows:

(1) The uncertain variables are assumed to have only positive deviations and (2) the uncertainty budgets are assumed to take only integer values. The robust model of the supply chain network can be written as follows:

$$
\begin{aligned}
& \operatorname{Min} \tilde{Z}=\sum_{a} t_{a}^{T} y_{a}+\sum_{b} t_{b}^{W} z_{b}+\sum_{g} \sum_{a} c_{g a}^{S T} x_{g a}+\sum_{a} \sum_{b} c_{a b}^{T W} u_{a b}+\sum_{b} \sum_{q} c_{b q}^{W M} v_{b q}
\end{aligned}
$$

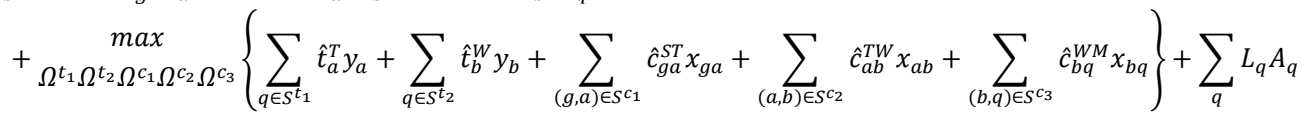

$\Omega^{t_{1}}=\left\{S^{t_{1}}\left|S^{t_{1}} \subseteq Q^{t_{1}},\right| S^{t_{1}} \mid \leq \Gamma^{t_{1}}\right\}$

$\Omega^{t_{2}}=\left\{S^{t_{2}}\left|S^{t_{2}} \subseteq Q^{t_{2}},\right| S^{t_{2}} \mid \leq \Gamma^{t_{2}}\right\}$

$\Omega^{c_{1}}=\left\{S^{c_{1}}\left|S^{c_{1}} \subseteq Q^{c_{1}},\right| S^{c_{1}} \mid \leq \Gamma^{c_{1}}\right\}$

$\Omega^{c_{2}}=\left\{S^{c_{2}}\left|S^{c_{2}} \subseteq Q^{c_{2}},\right| S^{c_{2}} \mid \leq \Gamma^{c_{2}}\right\}$

$\Omega^{c_{3}}=\left\{S^{c_{3}}\left|S^{c_{3}} \subseteq Q^{c_{3}},\right| S^{c_{3}} \mid \leq \Gamma^{c_{3}}\right\}$

$Q^{t_{1}}=\left\{a \mid \hat{t}_{a}^{T}>0\right\}, \Gamma^{t_{1}} \in\left[0,\left|Q^{t_{1}}\right|\right]$

$Q^{t_{2}}=\left\{b||_{b}^{W}>0\right\}, \Gamma^{t_{2}} \in\left[0,\left|Q^{t_{2}}\right|\right]$

$Q^{c_{1}}=\left\{(g, a) \mid \hat{c}_{g a}^{S T}>0\right\}, \Gamma^{c_{1}} \in\left[0,\left|Q^{c_{1}}\right|\right]$

$Q^{c_{2}}=\left\{(a, b) \mid \hat{c}_{a b}^{T W}>0\right\}, \Gamma^{c_{2}} \in\left[0,\left|Q^{c_{2}}\right|\right]$

$Q^{c_{3}}=\left\{(b, q) \mid \hat{c}_{b q}^{W M}>0\right\}, \Gamma^{c_{3}} \in\left[0,\left|Q^{c_{3}}\right|\right]$

Uncertainty in $t_{a}^{T}, t_{b}^{W}, c_{g a}^{S T}, c_{a b}^{T W}$, and $c_{b q}^{W M}$ :

Following the robust optimization procedure, linear protection functions are derived for the uncertain coefficients and the linear dual forms for the uncertain parameters are formulated. The nonlinear model can be transformed into the following linear model:

$$
\begin{gathered}
\operatorname{Min} \tilde{Z}=\sum_{a} t_{a}^{T} y_{a}+\sum_{b} t_{b}^{W} z_{b}+\sum_{g} \sum_{a} c_{g a}^{S T} x_{g a}+\sum_{a} \sum_{b} c_{a b}^{T W} u_{a b}+\sum_{b} \sum_{q} c_{b q}^{W M} v_{b q}+\lambda^{t_{1}} \Gamma^{t_{1}}+\sum_{g \in Q^{t}} \mu_{g}^{t_{1}}+\lambda^{t_{2}} \Gamma^{t_{2}}+\sum_{b \in Q^{t}} \mu_{b}^{t_{2}}+\lambda^{c_{1}} \Gamma^{c_{1}}+\sum_{(g, a) \in Q^{c_{1}}} \mu_{g a}^{c_{1}}+\lambda^{c_{2}} \Gamma^{c_{2}} \\
+\sum_{(a, b) \in Q^{c_{2}}} \mu_{a b}^{c_{2}}+\lambda^{c_{3}} \Gamma^{c_{3}}+\sum_{(b, q) \in Q^{c_{3}}}^{c_{b q}^{c_{3}}+\sum_{q} L_{q} A_{q}}
\end{gathered}
$$

The objective function is subject to the following constraints in its variables.

$\mu_{g a}^{c_{1}}+\lambda^{c_{1}} \geq \hat{c}_{g a}^{S T} x_{g a} \quad \forall(g, a) \in Q^{c_{1}}$

$\mu_{a b}^{c_{2}}+\lambda^{c_{2}} \geq \hat{c}_{a b}^{T W} u_{a b} \quad \forall(a, b) \in Q^{c_{2}}$

$\mu_{b q}^{c_{3}}+\lambda^{c_{3}} \geq \hat{c}_{b q}^{W M} v_{b q} \quad \forall(b, q) \in Q^{c_{3}}$

$\mu_{a}^{t_{1}}+\lambda^{t_{1}} \geq \hat{t}_{a}^{T} y_{a} \quad \forall a \in Q^{t_{1}}$

$\mu_{b}^{t_{2}}+\lambda^{t_{2}} \geq \hat{t}_{b}^{W} z_{b} \quad \forall b \in Q^{t_{2}}$

$\mu_{g a}^{c_{1}} \geq 0 \quad \forall(g, a) \in Q^{c_{1}}$ 
Citation: Raad Alzahrani, Mathematical Modeling of a Supply Chain with Uncertain Parameters. Australian Journal of Basic and Applied Sciences, $12(9)$ : 88-92. DOI: 10.22587/ajbas.2018.12.9.15

$\mu_{a b}^{c_{2}} \geq 0 \quad \forall(a, b) \in Q^{c_{2}}$

$\mu_{b q}^{c_{3}} \geq 0 \quad \forall(b, q) \in Q^{c_{3}}$

$\mu_{a}^{t_{1}} \geq 0 \quad \forall a \in Q^{t_{1}}, \mu_{b}^{t_{2}} \geq 0 \quad \forall b \in Q^{t_{2}}$

$\lambda^{c_{1}} \geq 0, \lambda^{c_{2}} \geq 0, \lambda^{c_{3}} \geq 0, \lambda^{t_{1}} \geq 0, \lambda^{t_{2}} \geq 0$

Uncertainty in $d_{q}, s_{g}$, and $L_{q}$

We define the auxiliary variable $H_{q}$ and add constraint (44) to the model:

$L_{q} A_{q} \leq H_{q} \quad \forall q$

$d+\frac{\Gamma^{m}}{m} \hat{d}-\sum_{b} v_{b q} \leq A_{q} \quad \forall q$

$\sum_{a} x_{g a}-s+\frac{\Gamma^{l}}{l} \hat{s} \leq S_{g} \quad \forall g$

$L_{q}\left(d+\frac{\Gamma^{m}}{m} \hat{d}-\sum_{b} v_{b q}\right)+\lambda_{q}^{p} \Gamma_{q}^{p}+\mu_{q}^{p} \leq H_{q} \quad \forall q$

$\mu_{q}^{p} \geq 0 \quad \forall q, \lambda_{q}^{p} \geq 0 \quad \forall q$

The model can be transformed to the following counterpart:

$$
\begin{aligned}
\operatorname{Min} \tilde{Z}=\sum_{a} t_{a}^{T} y_{a}+ & \sum_{b} t_{b}^{W} z_{b}+\sum_{g} \sum_{a} c_{g a}^{S T} x_{g a}+\sum_{a} \sum_{b} c_{a b}^{T W} u_{a b}+\sum_{b} \sum_{q} c_{b q}^{W M} v_{b q}+\lambda^{t_{1}} \Gamma^{t_{1}}+\sum_{g \in Q^{t}} \mu_{g}^{t_{1}}+\lambda^{t_{2}} \Gamma^{t_{2}}+\sum_{b \in Q^{t}} \mu_{b}^{t_{2}}+\lambda^{c_{1}} \Gamma^{c_{1}}+\sum_{(g, a) \in Q^{c_{1}}} \mu_{g a}^{c_{1}}+\lambda^{c_{2}} \Gamma^{c_{2}} \\
& +\sum_{(a, b) \in Q^{c_{2}}} \mu_{a b}^{c_{2}}+\lambda^{c_{3}} \Gamma^{c_{3}}+\sum_{(b, q) \in Q^{c_{3}}} \mu_{b q}^{c_{3}}+\sum_{q} H_{q}
\end{aligned}
$$

\section{RESULTS AND DISCUSSION}

The findings are derived from a comparison between the effects of variations in uncertain parameters on the objective function. For the same percentage of change in values of the uncertain parameters at the maximum conservatism degrees, variation in demand results in the greatest changes in the optimal value. For instance, $12 \%$ variation in demand contributes to $12.19 \%$ changes in the optimal costs. While, the same variation in supply, cost of locating manufacturers, cost of locating warehouses, cost of shipment from suppliers to manufacturers, cost of shipmen from manufacturers to warehouses, and cost of shipment from warehouses to markets result in $5.28,0.50,0.72,3.12,3.28$, and $4.46 \%$ changes in the optimal value of the objective function, respectively. The results for different variations in uncertain parameters when the conservatism degrees are fixed at their maxim levels are shown in Exhibit 2.

\begin{tabular}{|c|c|c|c|c|c|}
\hline & $5 \%$ & $12 \%$ & $18 \%$ & $25 \%$ & $30 \%$ \\
\hline Demand & 5.08 & 12.19 & 18.29 & 25.4 & 30.48 \\
\hline Supply & 2.2 & 5.28 & 7.92 & 11 & 13.2 \\
\hline Locating the manufacturers & 0.21 & 0.5 & 0.75 & 1.05 & 1.26 \\
\hline Locating warehouses & 0.3 & 0.72 & 1.08 & 1.5 & 1.8 \\
\hline Shipment (suppliers-manufacturers) & 1.3 & 3.12 & 4.68 & 6.5 & 7.8 \\
\hline Shipment (manufacturers-warehouses) & 1.37 & 3.28 & 4.93 & 6.85 & 8.22 \\
\hline Shipment (warehouses-market) & 1.86 & 4.64 & 6.69 & 9.3 & 11.16 \\
\hline
\end{tabular}

Exhibit 2: The effect of variation in uncertain parameters on the objective function.

This indicates that demand is the most significant factor and the management, in order to obtain the most cost effective supply chain, should focus on predictions of accurate demand. We analyze what happens when the uncertain parameters change by a range of relatively small to large rates and present a visual representation of the optimal solutions in each case to provide a real world application of the robust optimization approach to solving supply chain models with uncertain parameters.

\section{Conclusion:}

A four-layer supply chain network including multiple suppliers, manufacturers, warehouses, and markets is presented. The presented supply chain network involves uncertainties in the primary input parameters. A deterministic mathematical model of the supply chain is formulated. The deterministic model of the supply chain is transformed into a robust counterpart in order to handle the inherent uncertainties of the primary input parameters and obtain the optimal solutions. The objective of the robust model is to minimize the supply chain costs. The supply chain costs include fixed costs of locating manufacturers and warehouses as well as the variable costs of shipping raw materials from suppliers to manufacturers, shipping final products from manufacturers to warehouses, and shipping final products from warehouses to markets. The model is designed to incorporate the penalty costs in case demands of a certain market is not satisfied. Future work should examine the robust optimization of supply chain with multiple products. Robust optimization of a multi-objective model of supply chain networks, when the parameters are under uncertainty can be another future research direction. Our model can be extended depending on actual conditions. For instance, retailer as an additional layer of supply chain can be added to the model. It would also be worthwhile to examine the trade-off between the supply chain costs and environmental objectives such as minimizing releases of air contaminants caused by the fuels, which are consumed by the vehicles when shipping the products.

\section{REFERENCES}

Bertsimas, D., M. Sim, 2004. The price of robustness. Operations research, 52(1): 35-53.

Namdari, A., J. Drzymalski, H. Tohidi, 2017. Labor Productivity and Optimal Team Size in an Inspection Process. In IIE Annual Conference. Proceedings (pp: 854-859). Institute of Industrial and Systems Engineers (IISE).

Stadtler, H., 2008. Supply chain management-an overview. In Supply chain management and advanced planning (pp. 9-36). Springer, Berlin, Heidelberg.

Tohidi, H., A. Namdari, T.K. Keyser, J. Drzymalski, 2017. Information sharing systems and teamwork between sub-teams: a mathematical modeling perspective. Journal of Industrial Engineering International, 13(4): 513-520. 
Citation: Raad Alzahrani, Mathematical Modeling of a Supply Chain with Uncertain Parameters. Australian Journal of Basic and Applied Sciences, $12(9)$ : 88-92. DOI: $10.22587 /$ ajbas.2018.12.9.15

Vidal, C.J., M. Goetschalckx, 2001. A global supply chain model with transfer pricing and transportation cost allocation. European Journal of Operational Research, 129(1): 134-158.

Yue, D., F. You, 2014. Fair profit allocation in supply chain optimization with transfer price and revenue sharing: MINLP model and algorithm for cellulosic biofuel supply chains. AIChE Journal, 60(9): 3211-3229. 